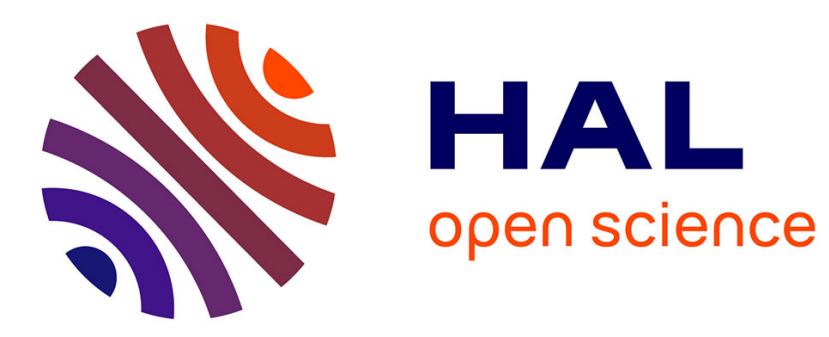

\title{
Insensitivity in processor-sharing networks
}

Thomas Bonald, Alexandre Proutière

\section{To cite this version:}

Thomas Bonald, Alexandre Proutière. Insensitivity in processor-sharing networks. Performance Evaluation, 2002. hal-01267145

\section{HAL Id: hal-01267145 \\ https://hal.science/hal-01267145}

Submitted on 4 Feb 2016

HAL is a multi-disciplinary open access archive for the deposit and dissemination of scientific research documents, whether they are published or not. The documents may come from teaching and research institutions in France or abroad, or from public or private research centers.
L'archive ouverte pluridisciplinaire HAL, est destinée au dépôt et à la diffusion de documents scientifiques de niveau recherche, publiés ou non, émanant des établissements d'enseignement et de recherche français ou étrangers, des laboratoires publics ou privés. 


\title{
Insensitivity in processor-sharing networks
}

\author{
T. Bonald and A. Proutière \\ France Telecom RधD \\ 38-40 rue du Général Leclerc, 92794 Issy-les-Moulineaux Cedex 9, France \\ \{Thomas.Bonald, Alexandre.Proutiere\}@francetelecom.com
}

\begin{abstract}
We consider an open network of processor-sharing nodes with state-dependent service capacities, i.e., the speed of each node may depend on the number of customers at any node. We demonstrate that the stationary distribution of the network state is insensitive to the distribution of service times if and only if the service capacities are balanced, i.e., the considered network is a Whittle network. The stationary distribution then has a closed-form expression and the expected sojourn time of a customer at any node is proportional to its required quantity of service. These results are extended to the cases of closed networks and state-dependent arrival rates and routing. Two simple examples illustrate the practical interest of these results in the context of communication networks.
\end{abstract}

Key words: Insensitivity, processor-sharing queue, Whittle networks, balance.

\section{Introduction}

Processor-sharing queues have traditionally been used to model so-called computer multi-access systems [15]. Typical examples include the processing capacity of a central processing unit (CPU) and the storage capacity of a database. More recently, processor-sharing queues have proved extremely successful in evaluating the mean transfer time of documents in data networks $[4,6-8,16]$. The service capacity then typically represents the bandwidth of a link which is assumed to be fairly shared between ongoing data flows $[4,8,16]$. More generally, networks of processor-sharing queues can be used to represent arbitrary network topologies, not just reduced to a single link [6,7]. The service capacity of a node then corresponds to the bandwidth allocated to ongoing flows on a particular route of the considered data network. As these flows must share the bandwidth of the corresponding links with all concurrent flows on this and other routes, the service capacity of a node generally depends on the number

Preprint submitted to Elsevier Science 19 June 2002 
of customers at each node: the corresponding model is a network of processorsharing queues with state-dependent service capacities, or "processor-sharing network" for short.

A key property of the processor-sharing queue is that, assuming Poisson arrivals, the stationary distribution of the number of customers does not depend on the distribution of service times [15]. This insensitivity property presents the practical interest in communication networks of allowing the development of engineering rules independently of precise traffic statistics. A typical example is the well-known insensitivity of the Erlang B formula which allows phone companies to dimension their network independently of the precise statistical characteristics of call durations. Similar robust dimensioning rules could be developed for data networks based on the insensitivity results for processorsharing networks derived in the cited work and further extended in the present paper. Insensitivity is a particularly desirable property in the context of the Internet where the nature of data traffic is continually evolving as new applications gain popularity (Web, peer-to-peer,...). It becomes sufficient for the network provider to just monitor overall load with no need to track the complex traffic characteristics of a panoply of different applications.

The insensitivity of queueing networks to the distribution of service times has received the attention of many authors [1-3,9,12,13,17,20,21,24,25]. A key result is that insensitivity is equivalent to partial balance for Markov processes [25] and generalized semi-Markov processes (GSMP) [20]. This provides a necessary and sufficient condition for closed networks to be insensitive to the service time distribution of each customer [21]. Insensitivity to the service time distribution at each node is not covered by this result, however. We must turn to more general results by Schassberger [21, Theorem 3.1] and Miyazawa [17, Theorem 4.1], who have proved the equivalence of partial balance and productform decomposability of the stationary distribution for general stochastic processes referred to as "scheme with relabelling" and "reallocatable GSMP", respectively. In particular, partial balance implies insensitivity for these types of process, but the converse is not true in general [17, Remark 5.1]. In the following we give a direct proof of a stronger result in the restricted framework of processor-sharing networks: insensitivity is equivalent to the balance of service capacities in this case, which is known to imply the partial balance of the corresponding Markov process [24]. Thus the balance of service capacities which was known to be a sufficient condition for insensitivity turns out to be also necessary. Whittle networks are the only insensitive processor-sharing networks.

We first describe the model and the balance property that characterizes Whittle networks. We then present the insensitivity results and some key performance results. These results are extended in the following two sections to the cases of closed queueing networks and state-dependent arrival rates and 
routing, respectively. We conclude the paper by two simple examples that illustrate the practical interest of these results in the context of communication networks.

\section{Processor-sharing networks}

We consider an open queueing network of $N$ nodes. At any node $i$, exogenous arrivals form a Poisson process of intensity $\nu_{i}$ and customers require i.i.d. exponentially distributed services of mean $1 / \mu_{i}$. The service discipline at each node is processor-sharing. After service completion at node $i$, a customer is routed to node $j$ with probability $p_{i j}$ and leaves the network with probability $p_{i} \equiv 1-\sum_{j} p_{i j}$. The effective arrival rate $\lambda_{i}$ at node $i$ is uniquely defined by the equations:

$$
\lambda_{i}=\nu_{i}+\sum_{j} \lambda_{j} p_{j i}, \quad i=1, \ldots, N
$$

We denote by $\rho_{i}=\lambda_{i} / \mu_{i}$ the traffic intensity at node $i$. The originality of the considered network is that the capacity (or speed) $\phi_{i}$ of node $i$ may depend on the state of the system $x=\left(x_{1}, \ldots, x_{N}\right)$, where $x_{i}$ is the number of customers at node $i$. We assume that $\phi_{i}(x)>0$ if and only if $x_{i}>0$. This queueing network will be referred to as a processor-sharing network.

Remark 1 (Feedback at nodes) Unless otherwise specified, we always assume that $p_{i i}=0$ for all $i=1, \ldots, N$. If $p_{i i}>0$ for some $i$, the state of the network is indeed the same as that of a network with service rates $\tilde{\mu}_{i}=\mu_{i}\left(1-p_{i i}\right)$ and routing probabilities $\tilde{p}_{i j}=p_{i j} /\left(1-p_{i i}\right)$ if $i \neq j$, and $\tilde{p}_{i i}=0$ otherwise.

The stochastic process $X=\left\{X_{t}, t \geq 0\right\}$ that describes the evolution of the number of customers at each node is a Markov process. Let $e_{i}$ be the unit vector with 1 in component $i$ and 0 elsewhere, for $i=1, \ldots, N$. When the network is in state $x$, the possible transitions are triggered by the movement of a customer from node $i$ to node $j$, in which case the next state is $T_{i}^{j} x \equiv x-e_{i}+e_{j}$, a departure from node $i$, in which case the next state is $T_{i} x \equiv x-e_{i}$, and an exogenous arrival at node $j$, in which case the next state is $T^{j} x \equiv x+e_{j}$. The balance equations that an invariant measure $\pi$ must satisfy are thus

$$
\begin{aligned}
& \pi(x) \sum_{i}\left(\nu_{i}+\phi_{i}(x) \mu_{i}\right)= \\
& \sum_{i} \pi\left(T_{i} x\right) \nu_{i}+\sum_{i, j} \pi\left(T_{i}^{j} x\right) \phi_{j}\left(T_{i}^{j} x\right) \mu_{j} p_{j i}+\sum_{i} \pi\left(T^{i} x\right) \phi_{i}\left(T^{i} x\right) \mu_{i} p_{i} .
\end{aligned}
$$

Note that, in view of Remark 1, equations (1) still hold in the presence of feedback. 


\section{Whittle networks}

A particular class of processor-sharing networks known as Whittle networks is characterized by the following balance property [24].

Definition 1 (Balance property) The capacities are said to be balanced if:

$$
\phi_{i}(x) \phi_{j}\left(T_{i} x\right)=\phi_{j}(x) \phi_{i}\left(T_{j} x\right), \quad i, j=1, \ldots, N, x_{i}>0, x_{j}>0 .
$$

Let $\left\langle x, T_{i_{1}} x, \ldots, T_{i_{n-1}} \ldots T_{i_{1}} x, 0\right\rangle$ be a direct path from state $x$ to state 0 , i.e., a path of length $n$ where $n$ is the number of customers in state $x$. The balance property implies that the expression

$$
\Phi(x)=1 / \phi_{i_{1}}(x) \phi_{i_{2}}\left(T_{i_{1}} x\right) \ldots \phi_{i_{n}}\left(T_{i_{n-1}} \ldots T_{i_{1}} x\right)
$$

is independent of the considered direct path. In particular, the capacities are uniquely characterized by the function $\Phi$, referred to as the balance function:

$$
\phi_{i}(x)=\frac{\Phi\left(T_{i} x\right)}{\Phi(x)}, \quad i=1, \ldots, N, x_{i}>0
$$

Conversely, if there exists a function $\Phi$ such that the capacities satisfy (3), it can be easily verified that these capacities are balanced. We say that the capacities are balanced by $\Phi$.

We have the following key result [24, Theorem 1.15].

Theorem 1 For a Whittle network, an invariant measure of $X$ is

$$
\pi(x)=\Phi(x) \prod_{i=1}^{N} \rho_{i}^{x_{i}}
$$

The proof simply consists in verifying that the measure $\pi$ satifies the partial balance equations

$$
\pi(x) \phi_{i}(x) \mu_{i}=\pi\left(T_{i} x\right) \nu_{i}+\sum_{j} \pi\left(T_{i}^{j} x\right) \phi_{j}\left(T_{i}^{j} x\right) \mu_{j} p_{j i}, \quad i=1, \ldots, N
$$

and

$$
\pi(x) \sum_{i} \nu_{i}=\sum_{i} \pi\left(T^{i} x\right) \phi_{i}\left(T^{i} x\right) \mu_{i} p_{i}
$$

Note again that, in view of Remark 1, equations (4)-(5) still hold in the presence of feedback. 


\section{Insensitivity}

From Theorem 1, Whittle networks are insensitive to the arrival rates, service rates and routing probabilities in that any invariant measure of $X$ depends on these quantities through the traffic intensities $\rho_{1}, \ldots, \rho_{N}$ only. Whittle networks are also known to be insensitive to the distribution of service times. For the class of Cox distributions which is known to form a dense subset within the set of all distributions of nonnegative random variables, this property is actually a direct consequence of the following simple result.

Proposition 2 Consider a processor-sharing network such that the total capacity of two nodes, say 1 and 2 , depends on the numbers of customers present at these nodes through their sum only and is equally shared between these customers, that is

$$
\frac{\phi_{1}(x)}{x_{1}}=\frac{\phi_{2}(x)}{x_{2}}=\frac{\phi_{1}(x)+\phi_{2}(x)}{x_{1}+x_{2}}, \quad x_{1}, x_{2}>0 .
$$

Then $\phi_{1}, \ldots, \phi_{N}$ are balanced by $\Phi$ if and only if $\phi_{1}+\phi_{2}, \phi_{3}, \ldots, \phi_{N}$ are balanced by $\tilde{\Phi}$, with

$$
\Phi(x)=\left(\begin{array}{c}
x_{1}+x_{2} \\
x_{1}
\end{array}\right) \tilde{\Phi}\left(x_{1}+x_{2}, x_{3}, \ldots, x_{N}\right) .
$$

Proof. The proof follows from (3).

Consider a Whittle network such that the total capacity of two nodes, say 1 and 2, depends on the numbers of customers present at these nodes through their sum only and is equally shared between these customers. In view of Proposition 2 and Theorem 1, an invariant measure $\pi$ of $X$ is given by

$$
\pi(x)=\left(\begin{array}{c}
x_{1}+x_{2} \\
x_{1}
\end{array}\right) \tilde{\Phi}\left(x_{1}+x_{2}, x_{3}, \ldots, x_{N}\right) \prod_{i=1}^{N} \rho_{i}^{x_{i}} .
$$

In particular, an invariant measure $\tilde{\pi}$ of the numbers of customers at nodes $1+2,3, \ldots, N$ is given by

$$
\tilde{\pi}\left(x_{1}+x_{2}, x_{3}, \ldots, x_{N}\right)=\tilde{\Phi}\left(x_{1}+x_{2}, x_{3}, \ldots, x_{N}\right)\left(\rho_{1}+\rho_{2}\right)^{x_{1}+x_{2}} \prod_{i=3}^{N} \rho_{i}^{x_{i}} .
$$

Now consider a Whittle network where the exponential distribution of service times at any node $i$ is replaced by a Cox distribution of $P_{i}$ phases with the same mean. This corresponds to a processor-sharing network with $P_{1}+\ldots+P_{N}$ nodes. From Proposition 2, this is still a Whittle network. In view of the previous property, the invariant measures of the total number of customers at any node $i$ of the initial network remain unchanged. We conclude that Whittle networks are indeed insensitive to the distribution of service times. 
Remark 3 Though we proved the result for the class of Cox distributions only, Whittle networks are known to be insensitive to any service time distribution. More general stochastic processes must then be considered [17,20].

It turns out that this insensitivity property uniquely characterizes Whittle networks among processor-sharing networks, as shown by the following result proved in Appendix A.

Theorem 2 A processor-sharing network is a Whittle network if and only if the invariant measures of $X$ remain unchanged when at any node $i$ and for any $\alpha_{i}, 0<\alpha_{i}<1$, customers require an exponentially distributed service of mean $1 / \alpha_{i} \times 1 / \mu_{i}$ with probability $\alpha_{i}$, a null service with probability $1-\alpha_{i}$.

The fact that the invariant measures of $X$ depend on the arrival rates, service rates and routing probabilities through the traffic intensities $\rho_{1}, \ldots, \rho_{N}$ only also characterizes Whittle networks among processor-sharing networks, as shown by the following two corollaries of Theorem 2. Corollary 2 is proved in Appendix B.

Corollary 1 A processor-sharing network with $p_{i j}=0$ for all $i, j$ is a Whittle network if and only if any invariant measure of $X$ depends on the arrival rates and service rates through their ratios $\rho_{1}, \ldots, \rho_{N}$ only.

Proof. Consider a processor-sharing network with $p_{i j}=0$ for all $i, j$. Assume that the invariant measures of $X$ remain unchanged when the arrival rate $\lambda_{i}$ and the service rate $\mu_{i}$ at any node $i$ are multiplied by a constant $\alpha_{i}, 0<$ $\alpha_{i}<1$. Noting that this modified network corresponds to the original network where at node $i$, customers require an exponentially distributed service of mean $1 / \alpha_{i} \times 1 / \mu_{i}$ with probability $\alpha_{i}$, a null service with probability $1-\alpha_{i}$, the proof follows from Theorem 2 .

Corollary 2 A processor-sharing network is a Whittle network if and only if the invariant measures of $X$ depend on the exogenous arrival rates and the routing probabilities through the effective arrival rates $\lambda_{1}, \ldots, \lambda_{N}$ only.

\section{Conditional expected sojourn time}

Consider a processor-sharing network. In the rest of the section we assume that the corresponding Markov process $X$ is positive recurrent and that $X$ is in steady state at time $t=0$. Denote by $S_{i}$ the sojourn time in node $i$ of the first customer arriving at node $i$. Applying Little's formula:

$$
E\left[x_{i}\right]=\lambda_{i} E_{i}\left[S_{i}\right],
$$


where $E_{i}$ is the expectation w.r.t. the Palm probability associated with the point process of arrivals at node $i$. The equivalent capacity of node $i$, defined as the mean required quantity of service at node $i$ divided by the expected sojourn time at node $i$, is then given by

$$
\gamma_{i} \equiv \frac{1 / \mu_{i}}{E_{i}\left[S_{i}\right]}=\frac{\rho_{i}}{E\left[x_{i}\right]}
$$

More generally, denote by $S_{I}$ the sojourn time in nodes $I$ of the first customer arriving at any node in $I$, where $I$ is a non-empty subset of $\{1, \ldots, N\}$. Applying Little's formula:

$$
\sum_{i \in I} E\left[x_{i}\right]=\lambda_{I} E_{I}\left[S_{I}\right]
$$

where $E_{I}$ is the expectation w.r.t. the Palm probability associated with the point process of arrivals at nodes $I$ and $\lambda_{I}$ is the arrival rate at nodes $I$ :

$$
\lambda_{I}=\sum_{i \in I}\left(\nu_{i}+\sum_{j \notin I} \lambda_{j} p_{j i}\right)
$$

Denote by $1 / \mu_{I}$ the mean required quantity of service at nodes $I$, given by $\lambda_{I} / \mu_{I}=\sum_{i \in I} \rho_{i}$. Similarly, define the equivalent capacity of nodes $I$ as the mean required quantity of service at nodes $I$ divided by the expected sojourn time at nodes $I$, namely:

$$
\gamma_{I} \equiv \frac{1 / \mu_{I}}{E_{I}\left[S_{I}\right]}=\frac{\sum_{i \in I} \rho_{i}}{\sum_{i \in I} E\left[x_{i}\right]} .
$$

Note that expressions (7)-(8) hold for general service time distributions.

Proposition 4 Consider a Whittle network such that the total capacity of nodes I depends on the numbers of customers present at these nodes through their sum only and is equally shared between these customers, that is

$$
\frac{\sum_{i \in I} \phi_{i}(x)}{\sum_{i \in I} x_{i}}=\frac{\phi_{i}(x)}{x_{i}}, \quad i \in I, x_{i}>0 .
$$

Then $\gamma_{I}=\gamma_{i}$ for all $i \in I$.

Proof. It follows from (6) that

$$
\frac{\rho_{i}}{E\left[x_{i}\right]}=\frac{\rho_{j}}{E\left[x_{j}\right]}, \quad \forall i, j \in I,
$$

so that $\gamma_{I}=\gamma_{i}$ for all $i \in I$. 
The following result shows that for Whittle networks, the expected sojourn time of a customer at any node is proportional to its required quantity of service.

Proposition 5 Consider a Whittle network. Denote by $\sigma_{i}$ the quantity of service required by the first customer arriving at node $i$. Then,

$$
\gamma_{i}=\frac{s}{E_{i}\left[S_{i} \mid \sigma_{i}=s\right]}, \quad \forall s>0
$$

Proof. We prove the result in the particular case where $\sigma_{i}$ takes a finite number of values. Replacing node $i$ by a set of nodes of equally shared overall capacity $\phi_{i}$, each node corresponding to a different value of $\sigma_{i}$, we obtain another Whittle network in view of Proposition 2. The proof then follows from Proposition 4.

\section{Closed networks}

In this section, we extend previous results to closed queueing networks, i.e., with a fixed total number of customers $M$ and $\nu_{i}=0$ for all $i$. Similar results hold for so-called mixed queueing networks. We first consider the case where routing is irreducible in the sense that each node is visited by the $M$ customers. Assume that the capacity of a particular node referred to as the "source", say node 0 , is a function $\psi_{0}$ of the number of customers at this node only. We have $\phi_{0}(x)=\psi_{0}(M-|x|)$, where $x=\left(x_{1}, \ldots, x_{N}\right)$ denoting the numbers of customers at any other node characterizes the system state and $|x|=\sum_{i=1}^{N} x_{i}$. The arrival "frequency" $\lambda_{i}$ at node $i$ is uniquely defined by the equations:

$$
\lambda_{i}=\sum_{j} \lambda_{j} p_{j i}, \quad i=0, \ldots, N,
$$

with $\sum_{i} \lambda_{i}=1$. We still define $\rho_{i}=\lambda_{i} / \mu_{i}$ for each node $i=0, \ldots, N$.

Consider the Markov process $X=\left\{X_{t}, t \geq 0\right\}$ describing the evolution of the numbers of customers at nodes $1, \ldots, N$. As the state space of $X$ is reduced to those vectors $x$ such that $|x|$ is less than or equal to $M, X$ is necessarily positive recurrent. The balance equations that an invariant measure $\pi$ must satisfy are simply

$$
\pi(x) \sum_{i} \phi_{i}(x) \mu_{i}=\sum_{i, j} \pi\left(T_{i}^{j} x\right) \phi_{j}\left(T_{i}^{j} x\right) \mu_{j} p_{j i}
$$

with the convention $T_{i}^{0} \equiv T_{i}$ for $i \neq 0, T_{0}^{j} \equiv T^{j}$ for $j \neq 0$, and $T_{0}^{0}$ is the identity operator. As for open networks, we say that a closed processor-sharing network 
is a Whittle network if the capacities $\phi_{1}, \ldots, \phi_{N}$ are balanced by a function $\Phi$. We have the equivalent of Theorem 1, which can also be proved by verifying that the measure $\pi$ satifies the partial balance equations for each node $i$ [24].

Theorem 3 For a closed irreducible Whittle network, an invariant measure of $X$ is

$$
\pi(x)=\Phi(x) \prod_{i=1}^{N} \rho_{i}^{x_{i}} \prod_{n=1}^{M-|x|} \frac{\rho_{0}}{\psi_{0}(n)}
$$

From Theorem 3, any invariant measure of $X$ depends on the routing probabilities through the arrival frequencies $\lambda_{1}, \ldots, \lambda_{N}$ only. In addition, it can be proved as in $\S 4$ that closed Whittle networks are insensitive to the distribution of service times. As for open networks, each of these properties characterizes Whittle networks among closed processor-sharing networks. Theorem 4 is proved in Appendix C. The proof of Corollary 3 is similar to that of Corollary 2 .

Theorem 4 A closed irreducible processor-sharing network is a Whittle network if and only if the invariant measures of $X$ remain unchanged when at any node $i \neq 0$ and for any $\alpha_{i}, 0<\alpha_{i}<1$, customers require an exponentially distributed service of mean $1 / \alpha_{i} \times 1 / \mu_{i}$ with probability $\alpha_{i}$, a null service with probability $1-\alpha_{i}$.

Corollary 3 A closed irreducible processor-sharing network is a Whittle network if and only if the invariant measures of $X$ depend on the routing probabilities through the arrival frequencies $\lambda_{1}, \ldots, \lambda_{N}$ only.

Finally, the performance results of $\S 5$ also apply in the context of closed networks, except that the effective arrival rate at any node $i$ is now given by:

$$
\frac{\sum_{x} \pi(x) \phi_{i}(x) \mu_{i}}{\sum_{x} \pi(x)}=\frac{\theta_{M}}{\theta_{M-1}} \lambda_{i},
$$

where $\theta_{M}$ denotes the normalization constant associated with the invariant measure $\pi$ given in Theorem 3 in the presence of $M$ customers.

Similar results hold when routing is reducible. Denote by $c_{1}, \ldots, c_{K}$ the $K$ subsets of nodes such that routing is irreducible on each of these subsets. Each set $c_{k}$ is visited by a fixed number of customers $M_{k}$. We assume that for each $k$, the capacity of a particular node $i_{k} \in c_{k}$ is a function $\psi_{k}$ of the number of customers at this node only. This node will be referred to as the "source" of set $c_{k}$. The arrival frequency $\lambda_{i}$ at any node $i$ of $c_{k}$ is uniquely defined by the equations:

$$
\lambda_{i}=\sum_{j \in c_{k}} \lambda_{j} p_{j i}, \quad i \in c_{k},
$$

with $\sum_{i \in c_{k}} \lambda_{i}=1$. We define $\varrho_{k}=\lambda_{i_{k}} / \mu_{i_{k}}$ and $\rho_{i}=\lambda_{i} / \mu_{i}$ for $i \in c_{k} \backslash i_{k}$. 
Denote by $N$ the number of nodes other than the sources, i.e., nodes in the set $\cup_{k}\left\{c_{k} \backslash i_{k}\right\}$. The system state $x=\left(x_{1}, \ldots, x_{N}\right)$ is uniquely characterized by the numbers of customers at these nodes only. If the capacities $\phi_{1}, \ldots, \phi_{N}$ are balanced by a function $\Phi$, an invariant measure $\pi$ of $X$ is

$$
\pi(x)=\Phi(x) \prod_{i=1}^{N} \rho_{i}^{x_{i}} \prod_{k=1}^{K} \prod_{n_{k}=1}^{M_{k}-\sum_{i \in c_{k} \backslash i_{k}} x_{i}} \frac{\varrho_{k}}{\psi_{k}\left(n_{k}\right)}
$$

Theorem 4 and Corollary 3 still hold in this case. The performance results of $\S 5$ also apply, except that the effective arrival rate at any node $i$ of $c_{k}$ is given by:

$$
\frac{\sum_{x} \pi(x) \phi_{i}(x) \mu_{i}}{\sum_{x} \pi(x)}=\frac{\theta_{M_{1}, \ldots, M_{k}, \ldots, M_{K}}}{\theta_{M_{1}, \ldots, M_{k}-1, \ldots, M_{K}}} \lambda_{i},
$$

where $\theta_{M_{1}, \ldots, M_{K}}$ denotes the normalization constant associated with the invariant measure $\pi$ given by (10) in the presence of $M_{1}, \ldots, M_{K}$ customers visiting the sets $c_{1}, \ldots, c_{K}$, respectively.

\section{State-dependent arrival rates and routing}

Another possible extension of the model is to consider state-dependent arrival rates $\nu_{i}(x)$ and routing probabilities $p_{i j}(x)$ so as to represent blocking phenomena for instance. Such networks have been studied in $[10,17,18,22,23]$. In the following we give a necessary and sufficient condition for these networks to be insensitive in the general case where the service capacities may be not balanced. To our knowledge, it covers all insensitive networks considered so far.

Remark 6 The class of insensitive processor-sharing networks with statedependent arrival rates and routing includes all restrictions of Whittle networks with reversible routing, $i . e .$, such that $\lambda_{i} p_{i j}=\lambda_{j} p_{j i}$ for all $i, j$. This follows from the reversibility of the corresponding Markov process $X$ [24]. Typical examples are so-called loss networks [14].

We consider open networks only, though similar results hold for closed networks. Assume that for any state $x$, the following equations have a unique solution $\lambda_{1}(x), \ldots, \lambda_{N}(x)$ :

$$
\lambda_{i}(x)=\nu_{i}(x)+\sum_{j} \lambda_{j}(x) p_{j i}\left(T^{j} x\right), \quad i=1, \ldots, N .
$$

We refer to $\rho_{i}(x)=\lambda_{i}(x) / \mu_{i}$ as the traffic intensity at node $i$ in state $x$. The balance equations that an invariant measure $\pi$ of the corresponding Markov process $X$ must satisfy are: 


$$
\begin{aligned}
& \pi(x) \sum_{i}\left(\nu_{i}(x)+\phi_{i}(x) \mu_{i}\right)=\sum_{i} \pi\left(T_{i} x\right) \nu_{i}\left(T_{i} x\right) \\
& +\sum_{i, j} \pi\left(T_{i}^{j} x\right) \phi_{j}\left(T_{i}^{j} x\right) \mu_{j} p_{j i}\left(T_{i}^{j} x\right)+\sum_{i} \pi\left(T^{i} x\right) \phi_{i}\left(T^{i} x\right) \mu_{i} p_{i}\left(T^{i} x\right) .
\end{aligned}
$$

Theorem 1 and Proposition 2 can be generalized as follows. Let $\psi_{i}$ be the function defined by

$$
\psi_{i}(x)=\frac{\rho_{i}\left(T_{i} x\right)}{\phi_{i}(x)}, \quad x_{i}>0
$$

Theorem 5 If $\psi_{1}, \ldots, \psi_{N}$ are balanced, an invariant measure of $X$ is given by $\pi(x)=\psi_{i_{1}}(x) \psi_{i_{2}}\left(T_{i_{1}} x\right) \ldots \psi_{i_{n}}\left(T_{i_{n-1}} \ldots T_{i_{1}} x\right)$, where $\left\langle x, T_{i_{1}} x, \ldots, T_{i_{n-1}} \ldots T_{i_{1}} x, 0\right\rangle$ is any path of length $n=\sum_{i} x_{i}$ from state $x$ to state 0 .

As for Theorem 1, the proof consists in verifying that the partial balance equations are satisfied for each node $i=1, \ldots, N$. When the functions $\psi_{1}, \ldots, \psi_{N}$ are balanced, $\pi(x)=0$ implies $\pi(x+y)=0$ for all $y \geq 0$. We thus implicitly restrict the analysis to those states $x$ such that $\pi(x)>0$. A balance function is then given by $\Psi=\pi^{-1}$.

Remark 7 If $\phi_{1}, \ldots, \phi_{N}$ are balanced by $\Phi, \psi_{1}, \ldots, \psi_{N}$ are balanced by $\Psi$ if and only if $\rho_{1}\left(T_{1} \cdot\right), \ldots, \rho_{N}\left(T_{N^{*}}\right)$ are balanced by $\Phi \times \Psi$.

Proposition 8 Assume that the total capacity of two nodes, say 1 and 2, depends on the numbers of customers present at these nodes through their sum only and is equally shared between these customers, that is

$$
\frac{\phi_{1}(x)}{x_{1}}=\frac{\phi_{2}(x)}{x_{2}}=\frac{\phi_{1}(x)+\phi_{2}(x)}{x_{1}+x_{2}}, \quad x_{1}, x_{2}>0 .
$$

Assume moreover that $\rho_{1}\left(T_{1} x\right)+\rho_{2}\left(T_{2} x\right)$ depends on $x_{1}, x_{2}$ through their sum only and that for some constants $w_{1}, w_{2}$,

$$
\frac{\rho_{1}\left(T_{1} x\right)}{w_{1}}=\frac{\rho_{2}\left(T_{2} x\right)}{w_{2}}=\frac{\rho_{1}\left(T_{1} x\right)+\rho_{2}\left(T_{2} x\right)}{w_{1}+w_{2}}, \quad x_{1}, x_{2}>0 .
$$

Then $\psi_{1}, \ldots, \psi_{N}$ are balanced by $\Psi$ if and only if $\frac{\rho_{1}\left(T_{1} \cdot\right)+\rho_{2}\left(T_{2} \cdot\right)}{\phi_{1}+\phi_{2}}, \psi_{3}, \ldots, \psi_{N}$ are balanced by $\tilde{\Psi}$, with

$$
\Psi(x)^{-1}=\left(\begin{array}{c}
x_{1}+x_{2} \\
x_{1}
\end{array}\right) \frac{w_{1}^{x_{1}} w_{2}^{x_{2}}}{\left(w_{1}+w_{2}\right)^{x_{1}+x_{2}}} \tilde{\Psi}\left(x_{1}+x_{2}, x_{3}, \ldots, x_{N}\right)^{-1} .
$$

Proof. The proof follows from the fact that

$$
\frac{\rho_{1}\left(T_{1} x\right)}{\phi_{1}(x)}=\frac{x_{1}+x_{2}}{x_{1}} \times \frac{w_{1}}{w_{1}+w_{2}} \times \frac{\rho_{1}\left(T_{1} x\right)+\rho_{2}\left(T_{2} x\right)}{\phi_{1}(x)+\phi_{2}(x)}, \quad x_{1}, x_{2}>0 .
$$


Applying the same reasoning as in $\S 4$, it follows from Proposition 8 that if the functions $\psi_{1}, \ldots, \psi_{N}$ are balanced, any invariant measure of $X$ is insensitive to the distribution of service times (at least for Cox distributions). Conversely, this insensitivity property implies the balance of the functions $\psi_{1}, \ldots, \psi_{N}$, as shown by the following generalization of Theorem 2 proved in Appendix D. Corollaries 1 and 2 can be generalized in the same way.

Theorem 6 The functions $\psi_{1}, \ldots, \psi_{N}$ are balanced if and only if the invariant measure of $X$ remain unchanged when at any node $i$ and for any $\alpha_{i}, 0<\alpha_{i}<$ 1 , customers require an exponentially distributed service of mean $1 / \alpha_{i} \times 1 / \mu_{i}$ with probability $\alpha_{i}$, a null service with probability $1-\alpha_{i}$.

Theorem 6 allows to characterize insensitive processor-sharing networks with state-dependent arrival rates and routing probabilities. When the Markov process $X$ is positive recurrent, the expected sojourn time of a customer at any node is proportional to its required quantity of service. The proof follows from Proposition 8 as in $\S 5$.

\section{Application to communication networks}

Processor-sharing networks provide a very useful and powerful tool to evaluate flow-level performance of communication networks. The model of $\S 2$ is sufficiently general to represent any characteristics of real traffic, including the fact that flows are generally generated within sessions, each session being composed of an alterning series of flows and "think-time" [4,7]. Closed networks are appropriate for modeling access networks where the user population is typically limited, while open networks can be used to model backbone networks where the user population is so large that sessions typically arrive as Poisson processes. Applying the results of $\S 4-6$, it is possible to characterize those bandwidth allocations that guarantee the insensitivity of network performance to all traffic characteristics, including the distribution of the number of flows per session, flow size and think-time duration distributions and possible correlations between successive flows [7]. In this section we give two examples assuming for simplicity that flows arrive as Poisson processes, though the derived results do not depend on this assumption.

\subsection{A single link with multiple rate limits}

Consider a single unit capacity link shared by $N$ types of flow. Flows of type $i$ are characterized by a rate limit $a_{i}$ typically representing external constraints such as the user's modem speed. Let $x=\left(x_{1}, \ldots, x_{N}\right)$, where $x_{i}$ is the number 
of ongoing flows of type $i$. The bandwidth $\phi_{i}(x)$ allocated to flows of type $i$ must satisfy the constraints:

$$
\sum_{i} \phi_{i}(x) \leq 1 \quad \text { and } \quad \phi_{i}(x) \leq a_{i} x_{i}, \quad i=1, \ldots, N
$$

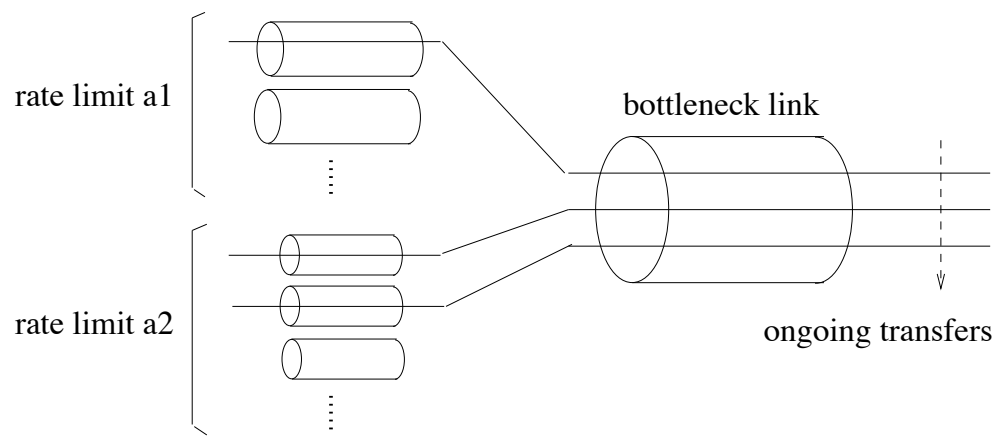

Fig. 1. A link with two distinct rate limits

The bandwidth allocation is insensitive if and only if the functions $\phi_{i}$ are balanced. It can actually been shown that there is a unique efficient insensitive allocation, i.e., such that

$$
\sum_{i=1}^{N} \phi_{i}(x)=\max (a . x, 1), \quad \text { a.x } \equiv \sum_{i=1}^{N} a_{i} x_{i} .
$$

This allocation is characterized by the balance function $\Phi$ defined by

$$
\begin{gathered}
\Phi(x)=\prod_{i} \frac{1}{a_{i}^{x_{i}} x_{i} !} \quad \text { if } a \cdot x \leq 1, \\
\Phi(x)=\sum_{y \in A(x)}\left(\begin{array}{c}
\sum_{i}\left(x_{i}-y_{i}\right)-1 \\
\sum_{i \notin I(y)}\left(x_{i}-y_{i}\right)
\end{array}\right)\left(\begin{array}{c}
\sum_{i \notin I(y)}\left(x_{i}-y_{i}\right) \\
\left(x_{i}-y_{i}\right), i \notin I(y)
\end{array}\right)\left(\begin{array}{c}
\sum_{i \in I(y)}\left(x_{i}-y_{i}\right) \\
\left(x_{i}-y_{i}\right), i \in I(y)
\end{array}\right) \prod_{i} \frac{1}{a_{i}^{y_{i}} y_{i} !}
\end{gathered}
$$

otherwise, where $A(x)=\{y, y \leq x, a . y \leq 1, I(y) \neq \emptyset\}$ and $I(y)$ denotes the set of indices $\left\{j, a_{j}+a . y>1\right\}$. The stationary distribution of the number of flows of each type immediately follows in view of Theorem 1 . The resulting throughput, defined as the ratio of the flow size to the mean flow duration, is given by (7) for each type of flow.

Note that this insensitive allocation differs from the max-min allocation $[5,6]$ except when there is a single type of flow. The model then corresponds to a multi-server processor-sharing queue which is indeed known to be insensitive $[4,11]$. Figure 2 compares the throughput obtained with both allocations in case of two rate limits $a_{1}=0.5$ and $a_{2}=0.2$ with respect to the overall traffic intensity $\rho_{1}+\rho_{2}$. The traffic intensity is the same for each type of flow. The results for the max-min allocation are obtained by simulation with Poisson flow arrivals and i.i.d. exponentially distributed flow sizes. 


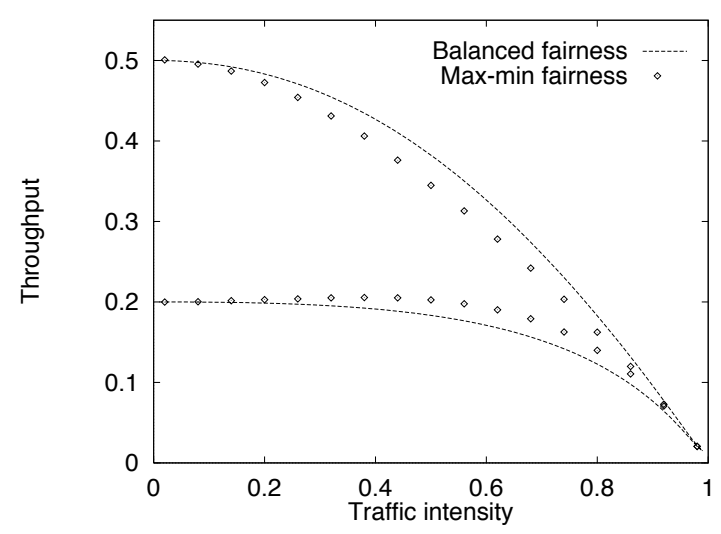

Fig. 2. Throughput evaluation in case of two rate limits $\left(a_{1}=0.5, a_{2}=0.2\right)$

\subsection{Parallel links with adaptive routing}

Consider a set of $L$ parallel links of respective capacities $C_{1}, \ldots, C_{L}$. Flows of mean size $1 / \mu$ arrive according to a Poisson process of intensity $\lambda$, generating a traffic intensity $\rho=\lambda / \mu$. Each flow is routed to one of the $L$ links. Let $x=\left(x_{1}, \ldots, x_{L}\right)$, where $x_{i}$ is the number of ongoing flows on link $i$. We envisage an adaptive routing, i.e., the routing decision is performed flow by flow and depends on the network state [19]. Denote by $\nu_{i}(x)$ the resulting flow arrival rate at link $i$ when the network is in state $x: \nu_{i}(x) / \lambda$ is the probability an arriving flow is routed over link $i$ in state $x$. The traffic intensity at link $i$ is then given by $\rho_{i}(x)=\nu_{i}(x) / \mu$.

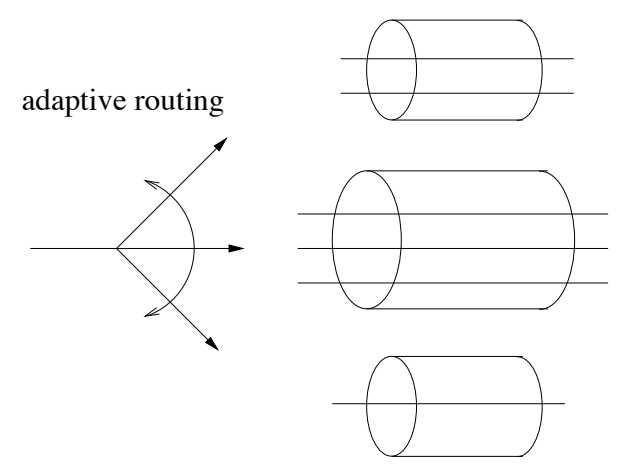

Fig. 3. Parallel links with adaptive routing

Assume that a maximum of $N_{i}$ flows can be simultaneously served by link $i$, so that a minimum rate of $C_{i} / N_{i}$ is guaranteed to all flows on this link. Assume further that the routing decision is such that a flow is rejected if and only if all links are saturated, that is

$$
\sum_{i, x_{i}<N_{i}} \nu_{i}(x) / \lambda=1, \quad \text { for all } x \text { such that } x_{i}<N_{i} \text { for some } i .
$$

In view of Theorem 6 and Remark 7, the routing algorithm is insensitive if and 
only if the traffic intensities are balanced. With the previous assumptions, it can be easily shown that such an insensitive routing algorithm is unique and is characterized by:

$$
\nu_{i}(x)=\frac{N_{i}-x_{i}}{\sum_{j=1}^{L}\left(N_{j}-x_{j}\right)} \lambda .
$$

An invariant measure of the corresponding Markov process $X$ is then given by:

$$
\pi(x)=\left(\begin{array}{c}
\sum_{j=1}^{L}\left(N_{j}-x_{j}\right) \\
N_{1}-x_{1}, \ldots, N_{L}-x_{L}
\end{array}\right) \prod_{i=1}^{L}\left(\frac{\rho}{C_{i}}\right)^{x_{i}} .
$$

We obtain the blocking probability:

$$
P=\frac{\pi\left(N_{1}, \ldots, N_{L}\right)}{\sum_{i=1}^{L} \sum_{x_{i}=0}^{N_{i}} \pi\left(x_{1}, \ldots, x_{L}\right)} .
$$

Figure 4 compares the blocking probability obtained with different routing strategies in case of two parallel unit capacity links with $N_{1}=N_{2}=10$. "Static routing" refers to a non-adaptive strategy where half of the flows are routed to one link, half to the other link. "Insensitive routing" refers to the adaptative strategy defined by (12). "Greedy routing" refers to an adaptive strategy where flows are routed to that link $i$ with the highest potential rate $C_{i} /\left(x_{i}+1\right)$, as suggested in [19]. This routing algorithm is sensitive as it differs from (12). The corresponding results are thus obtained by simulation using Poisson flow arrivals and i.i.d.exponentially distributed flow sizes. Figure 4 shows that expression (13) provides an accurate and conservative estimation of the resulting blocking probability.

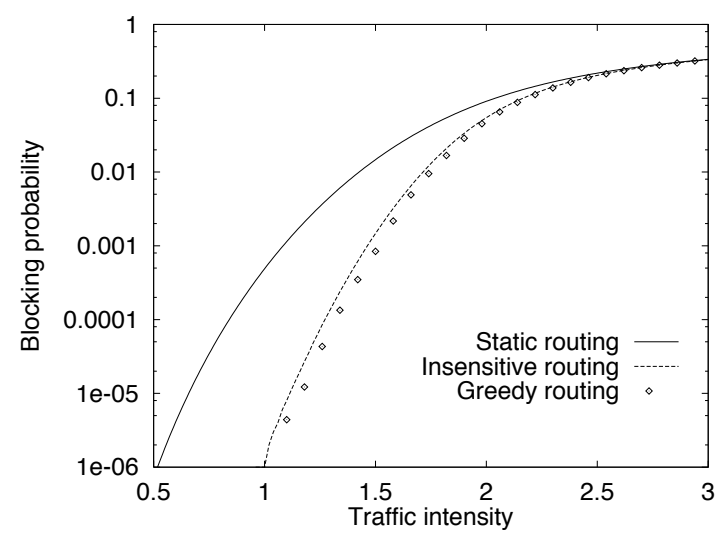

Fig. 4. Blocking probability for different routing strategies 


\section{Appendix}

\section{A Proof of Theorem 2}

Consider a processor-sharing network. In view of Remark 1, we can assume without loss of generality that there is no feedback, that is $p_{i i}=0$ for all $i=$ $1, \ldots, N$. When the service required at node 1 is changed into an exponentially distributed service of mean $1 / \alpha_{1} \times 1 / \mu_{1}$ with some probability $\alpha_{1}>0$ or a null service with probability $1-\alpha_{1}$, the state of the network is that of the processor-sharing network defined by exogenous arrival rates $\tilde{\nu}_{1}=\nu_{1} \alpha_{1}$, $\tilde{\nu}_{i}=\nu_{i}+\nu_{1}\left(1-\alpha_{1}\right) p_{1 i}$ for $i \neq 1$, service rates $\tilde{\mu}_{1}=\mu_{1} \alpha_{1}, \tilde{\mu}_{i}=\mu_{i}$ for $i \neq 1$, and routing probabilities $\tilde{p}_{1 i}=p_{1 i}, \tilde{p}_{i 1}=p_{i 1} \alpha_{1}, \tilde{p}_{i j}=p_{i j}+p_{i 1}\left(1-\alpha_{1}\right) p_{1 j}$ for $i, j \neq 1$. Now assume the original network is a Whittle network. Any invariant measure $\pi$ satisfies the partial balance equations (4)-(5). It can then be easily verified that $\pi$ also satisfies the partial balance equations (4)-(5) for the new network.

Conversely, consider a processor-sharing network such that the corresponding invariant measures remain unchanged when at node 1 and for any $\alpha_{1}, 0<\alpha_{1}<$ 1 , customers require an exponentially distributed service of mean $1 / \alpha_{1} \times 1 / \mu_{1}$ with probability $\alpha_{1}$, a null service with probability $1-\alpha_{1}$. Letting $\alpha_{1}$ tend to zero in the corresponding balance equations (1), we obtain

$$
\begin{aligned}
& \pi(x) \sum_{i \neq 1}\left(\phi_{i}(x) \mu_{i}+\tilde{\nu}_{i}\right)= \\
& \sum_{i \neq 1} \pi\left(T_{i} x\right) \tilde{\nu}_{i}+\sum_{i, j \neq 1} \pi\left(T_{i j} x\right) \phi_{j}\left(T_{i j} x\right) \mu_{j} \tilde{p}_{j i}+\sum_{i \neq 1} \pi\left(T^{i} x\right) \phi_{i}\left(T^{i} x\right) \mu_{i} \tilde{p}_{i}
\end{aligned}
$$

where $\tilde{\nu}_{i}=\nu_{i}+\nu_{1} p_{1 i}, \tilde{p}_{i j}=p_{i j}+p_{i 1} p_{1 j}$ and $\tilde{p}_{i}=1-\sum_{j \neq 1} \tilde{p}_{i j}, i, j \neq 1$. This shows that for any fixed $x_{1}, \pi\left(x_{1}, \cdot\right)$ is an invariant measure for the processorsharing network consisting of nodes $2, \ldots, N$ only with state-dependent capacities $\phi_{2}\left(x_{1}, \cdot\right), \ldots, \phi_{N}\left(x_{1}, \cdot\right)$, which corresponds to the original network when node 1 is removed. Note that some feedback may have been generated, which can again be eliminated in view of Remark 1 .

Now consider a processor-sharing network such that the corresponding invariant measures remain unchanged when at any node $i=1, \ldots, N-1$ and for any $\alpha_{i}, 0<\alpha_{i}<1$, customers require an exponentially distributed service of mean $1 / \alpha_{i} \times 1 / \mu_{i}$ with probability $\alpha_{i}$, a null service with probability $1-\alpha_{i}$. Applying successively the previous property, we conclude that for any fixed $x_{1}, \ldots, x_{N-1}$, $\pi\left(x_{1}, \ldots, x_{N-1}, \cdot\right)$ is an invariant measure for the processor-sharing network consisting of node $N$ only with state-dependent capacity $\phi_{N}\left(x_{1}, \ldots, x_{N-1}, \cdot\right)$, which corresponds to the original network when nodes $1, \ldots, N-1$ are re- 
moved. The balance equations for this particular network are simply

$$
\begin{aligned}
& \pi(x)\left(\phi_{N}(x) \mu_{N}+\hat{\nu}_{N}\right)= \\
& \pi\left(T_{N} x\right) \hat{\nu}_{N}+\pi(x) \phi_{N}(x) \mu_{N} \hat{p}_{N N}+\pi\left(T^{N} x\right) \phi_{N}\left(T^{N} x\right) \mu_{N}\left(1-\hat{p}_{N N}\right),
\end{aligned}
$$

where $\hat{p}_{N N}$ is the probability that a customer comes back at node $N$ after service completion and $\hat{\nu}_{N}=\lambda_{N}\left(1-\hat{p}_{N N}\right)$. These are the balance equations of a birth and death process. In particular, $\pi(x) \phi_{N}(x)=\pi\left(T_{N} x\right) \rho_{N}$. As all nodes are equivalent, we conclude that

$$
\pi(x) \phi_{i}(x)=\pi\left(T_{i} x\right) \rho_{i}, \quad i=1, \ldots, N .
$$

Thus the capacities $\phi_{i}$ are balanced by the function $\Phi$ defined by

$$
\Phi(x)=\frac{\pi(x)}{\prod_{i=1}^{N} \rho_{i}^{x_{i}}} .
$$

The considered network is a Whittle network.

\section{B Proof of Corollary 2}

Consider a processor-sharing network without feedback at any node. Assume that the corresponding invariant measures depend on the exogenous arrival rates and the routing probabilities through the effective arrival rates $\lambda_{1}, \ldots, \lambda_{N}$ only. For any fixed $\alpha_{1}$, with $0<\alpha_{1}<1$, these invariant measures remain unchanged when the exogenous arrival rates are $\tilde{\nu}_{1}=\alpha_{1} \nu_{1}, \tilde{\nu}_{i}=\nu_{i}+\nu_{1}(1-$ $\left.\alpha_{1}\right) p_{1 i}$ for $i \neq 1$, and the routing probabilities are $\tilde{p}_{11}=1-\alpha_{1}, \tilde{p}_{1 i}=p_{1 i} \alpha_{1}$ and $\tilde{p}_{i 1}=p_{i 1} \alpha_{1}$ for $i \neq 1, \tilde{p}_{i j}=p_{i j}+p_{i 1}\left(1-\alpha_{1}\right) p_{1 j}$ for $i, j \neq 1$. It can indeed be easily verified that the effective arrival rates remain unchanged with these new exogenous arrival rates and routing probabilities. In view of Remark 1, this new network corresponds to the original network where at node 1 , customers require an exponentially distributed service of mean $1 / \alpha_{1} \times 1 / \mu_{1}$ with probability $\alpha_{1}$, a null service with probability $1-\alpha_{1}$. The proof then follows from Theorem 2 .

\section{Proof of Theorem 4}

Consider a closed Whittle network. We proved as for open networks that the invariant measures of $X$ remain unchanged when at any node $i$ and for any $\alpha_{i}, 0<\alpha_{i}<1$, customers require an exponentially distributed service of mean $1 / \alpha_{i} \times 1 / \mu_{i}$ with probability $\alpha_{i}$, a null service with probability $1-\alpha_{i}$. 
Conversely, consider a processor-sharing network which satisfies this property. As in the proof of Theorem 2, we prove that for any fixed $x_{1}, \ldots, x_{N-1}$, $\pi\left(x_{1}, \ldots, x_{N-1}, \cdot\right)$ is an invariant measure for the closed processor-sharing network consisting of the source and node $N$ with state-dependent capacity $\phi_{N}\left(x_{1}, \ldots, x_{N-1}, \cdot\right)$ only, which corresponds to the original network when nodes $1, \ldots, N-1$ are removed. The balance equations for this particular network are simply

$$
\begin{aligned}
& \pi(x)\left(\phi_{0}(x) \mu_{0}\left(1-\hat{p}_{00}\right)+\phi_{N}(x) \mu_{N}\left(1-\hat{p}_{N N}\right)\right)= \\
& \pi\left(T_{N} x\right) \phi_{0}\left(T_{N} x\right) \mu_{0}\left(1-\hat{p}_{00}\right)+\pi\left(T^{N} x\right) \phi_{N}\left(T^{N} x\right) \mu_{N}\left(1-\hat{p}_{N N}\right),
\end{aligned}
$$

where $\hat{p}_{00}$ and $\hat{p}_{N N}$ are the feedback probabilities at nodes 0 and $N$, respectively. These are the balance equations of a birth and death process. Noting that $\lambda_{0} / \lambda_{N}=\left(1-\hat{p}_{00}\right) /\left(1-\hat{p}_{N N}\right)$, we conclude that

$$
\pi(x) \phi_{N}(x) \rho_{0}=\pi\left(T_{N} x\right) \phi_{0}\left(T_{N} x\right) \rho_{N}
$$

As nodes $1, \ldots, N$ are equivalent, we have

$$
\pi(x) \phi_{i}(x) \rho_{0}=\pi\left(T_{i} x\right) \phi_{0}\left(T_{i} x\right) \rho_{i}, \quad i=1, \ldots, N
$$

Thus the capacities $\phi_{1}, \ldots, \phi_{N}$ are balanced by the function $\Phi$ defined by

$$
\Phi(x)=\frac{\pi(x)}{\prod_{i=1}^{N} \rho_{i}^{x_{i}}} \prod_{i=1}^{M-|x|} \frac{\psi_{0}(n)}{\rho_{0}} .
$$

The considered network is a Whittle network.

\section{Proof of Theorem 6}

The proof is the same as that of Theorem 2, except that when the service required at node 1 is changed into an exponentially distributed service of mean $1 / \alpha_{1} \times 1 / \mu_{1}$ with some probability $\alpha_{1}>0$ or a null service with probability $1-\alpha_{1}$, the new processor-sharing network is characterized by state-dependent arrival rates $\tilde{\nu}_{1}(x)=\alpha_{1} \nu_{1}(x)$ and $\tilde{\nu}_{i}(x)=\nu_{i}(x)+\nu_{1}(x)\left(1-\alpha_{1}\right) p_{1 i}\left(T^{1} x\right)$ for $i \neq 1$, service rates $\tilde{\mu}_{1}=\alpha_{1} \mu_{1}$ and $\tilde{\mu}_{i}=\mu_{i}$ for $i \neq 1$, and state-dependent routing probabilities $\tilde{p}_{1 i}(x)=p_{1 i}(x), \tilde{p}_{i 1}(x)=\alpha_{1} p_{i 1}(x)$ and $\tilde{p}_{i j}(x)=p_{i j}(x)+$ $p_{i 1}(x)\left(1-\alpha_{1}\right) p_{1 j}\left(T_{i}^{1} x\right)$ for $i, j \neq 1$. 


\section{References}

[1] F. Baccelli and P. Brémaud, Palm probability and stationary queues, Lectures notes in Statistics 41 (1987).

[2] A.D. Barbour, Generalized semi-Markov schemes and open queueing networks, Journal of Applied Probability 19 (1982) 469-474.

[3] F. Baskett, K.M. Chandy, R.R. Muntz and F.G. Palacios, Open, closed and mixed networks of queues with different classes of customers, J. Assoc. Comput. Mach. 22 (1975) 248-260.

[4] S. Ben Fredj, T. Bonald, A. Proutière, G. Regnié, J.W. Roberts, Statistical bandwidth sharing: A study of congestion at flow level, in: Proceedings of $A C M$ SIGCOMM 2001.

[5] D. Bertsekas and R. Gallager, Data Networks (Prentice Hall, 1987).

[6] T. Bonald and L. Massoulié, Impact of fairness on Internet performance, in: Proceedings of ACM SIGMETRICS 2001.

[7] T. Bonald and A. Proutière, Insensitive bandwidth sharing, in: Proceedings of IEEE GLOBECOM 2002.

[8] T. Bonald, A. Proutière, G. Régnié, J.W. Roberts, Insensitivity results in statistical bandwidth sharing, in: Proceedings of ITC 17 (Elsevier, 2001).

[9] D. Burman, Insensitivity in queueing systems, Advances in Applied Probability 13 (1981) 846-859.

[10] X. Chao, M. Miyazawa, R. F. Serfozo and H. Takada, Markov network processes with product form stationary distributions, Queueing Systems 28 (1998) 377401.

[11] J. W. Cohen, The multiple phase service network with generalized processor sharing, Acta Informatica 12 (1979) 245-284.

[12] W. Henderson, Insensitivity and reversed Markov processes, Advances in Applied Probability 15 (1983) 752-768.

[13] F.P. Kelly, Reversibility and Stochastic Networks (Wiley, 1979).

[14] F.P. Kelly, Loss networks, Annals of Applied Probability 1 (1991) 319-378.

[15] L. Kleinrock, Queueing Systems, Volume 2 (J. Wiley \& Sons, 1975).

[16] L. Massoulié and J.W. Roberts, Bandwidth sharing and admission control for elastic traffic, Telecommunication Systems 15 (2000) 185-201.

[17] M. Miyazawa, Insensitivity and product-form decomposability of reallocatable GSMP, Advances in Applied Probability 25 (1993) 415-437.

[18] M. Miyazawa and H. Takada, Traffic flows and product form solutions in stochastic transfer networks, Queueing Systems 37 (2001) 199-232. 
[19] S. Oueslati Boulahia and E. Oubagha, An approach to elastic flow routing, in: Proceedings of ITC 16 (Elsevier, 1999).

[20] R. Schassberger, Insensitivity of steady state distributions of generalized semiMarkov processes with speeds, Advances in Applied Probability 10 (1978) 836851 .

[21] R. Schassberger, Two remarks on insensitive stochastic models, Advances in Applied Probability 18 (1986) 791-814.

[22] R.F. Serfozo, Markovian network processes: Congestion-dependent routing and processing, Queueing Systems 5 (1989) 5-36.

[23] R. F. Serfozo, Queueing networks with dependent nodes and concurrent movements, Queueing Systems 13 (1993) 143-182.

[24] R.F. Serfozo, Introduction to Stochastic Networks (Springer Verlag, 1999).

[25] P. Whittle, Partial balance and insensitivity, Journal of Applied Probability 22 (1985) 168-176. 